\title{
Antiproton and antilambda annihilations on several nucleons
}

\author{
J. Cugnon and J. Vandermeulen \\ Université de Liege, Physique Nucléaire Théorique, Institut de Physique au Sart Tilman, B.5, B-4000 Liege 1, Belgium
}

(Received 12 August 1988)

\begin{abstract}
Antiproton and antilambda annihilations involving several nucleons at the same time are assumed and arguments in favor of such processes are discussed. A statistical model for annihilation on one or several nucleons is studied. It is shown to give very good results for annihilation on one nucleon. The predictions of such a model for annihilation on several nucleons are discussed extensively. The most important of those is the enhancement of the strange particle yield. Other predictions are made for remarkable two-body channels and in particular for the mesonless annihilation $\bar{n}^{3} \mathrm{He} \rightarrow p p$. A geometrical model is made to estimate the probability of having annihilation on several nucleons in nuclei. Finally, the relationship between our statistical approach and current dynamical models is discussed.
\end{abstract}

\section{INTRODUCTION}

The possibility of annihilating an antiproton on more than one nucleon in atomic nuclei, suggested already a long time ago, ${ }^{1}$ has received more and more attention in recent years, ${ }^{2-4}$ especially because of the appearance of new data ${ }^{5-7}$ that seem to require this phenomenon. Furthermore, in previous papers, ${ }^{4,8}$ we tried to single out the main features of a two-nucleon annihilation process using a simple statistical approach and showed that strangeness production is enhanced compared to what it is for antinuclon-nucleon annihilation. Here we give more detail about the model and discuss new features. Actually, our purpose is fourfold. First, we want to extend it to $B>1$ annihilations (here $B$ represents the baryon number of the annihilating system), paying attention to strangeness production. Second, we discuss the relative probability of the various $B$ values and study a simplified geometric model for estimating this probability. Third, we make specific predictions for two-body final states, in particular for $\bar{n}^{3} \mathrm{He} \rightarrow p p$. Fourth, we investigate properties of $B=0$ as well as $B>0$ antilambda annihilations.

The basic premise of our approach is that antiproton annihilation is a complicated process, which, therefore, can be handled by a statistical approach. It has been known for a long time that the main features of antiproton-proton annihilations at rest and at low momentum (especially the yields of the pionic final states) are largely consistent with such an assumption. ${ }^{9-13} \mathrm{Al}$ though it is clear that there exists some deviations from the statistical picture in antiproton-proton annihilation, ${ }^{14}$ it is certainly interesting to see what are the predictions of the statistical approach to $B>0$ annihilations. Ultimately, the predictions may be used to reveal the existence of $B>0$ annihilations and possibly to evaluate their frequency.

The paper is organized as follows. Section II contains a description of the statistical model that we used and of its main predictions and features. It is a microcanonical approach in the sense that energy and baryon number are conserved exactly. In Sec. III, we discuss the relative probabilities for various values of $B$ in a given nucleus. We study a simple geometric model to estimate these probabilities and single out the main physical parameters which determine these probabilities. In Sec. IV, we present our estimate for remarkable decay channels, like two-body final states. Section $\mathrm{V}$ presents an extension of our approach to antilambda annihilations. Finally, Sec. VI contains a discussion of the model and its relationship to more dynamical approaches.

\section{THE STATISTICAL MODEL}

For $B=0$ annihilations, our statistical model ${ }^{4}$ is embodied by the following frequency distributions for, respectively, $n$-pion and $(K \bar{K}+l)$-pion events:

$$
\begin{aligned}
& f_{0}(n \pi)=A_{0}\left(\lambda C_{0}\right)^{n-1} R_{n}\left(\sqrt{s} ; n m_{\pi}\right), n \geq 2, \\
& f_{0}(K \bar{K}, l \pi) \\
& \quad=A_{0} \beta_{S}\left(\lambda_{S} C_{0}\right)^{l+1} R_{l+2}\left(\sqrt{s} ; 2 m_{k}, l m_{\pi}\right), \quad l \geq 0 .
\end{aligned}
$$

In these equations, $R_{i}$ is the statistical bootstrap phase space integral. ${ }^{15}$ The dimensional constant $C_{0}=\left(4 \pi m_{\pi}\right)^{-2}$ plays a role similar to the interaction volume in the original Fermi statistical model. ${ }^{16}$ The normalization constant $A_{0}$ ensures that all the frequencies sum up to unity. The free parameter $\lambda$ is directly related to the average value of $n$. It should be close to one if the interacting system has a typical hadronic linear dimension, i.e., $\sim m_{\pi}^{-1}$. It turned out to be necessary to introduce two additional free parameters, $\lambda_{S}$ and $\beta_{S}$ to reproduce the fine detail of strangeness production. Roughly speaking, $\beta_{S}$, that we call the hindrance factor, determines the relative yield of kaonic to pionic annihilation, and $\lambda_{S}$ is moderately dependent upon $\langle l\rangle$ (in Ref. 4, $\lambda_{S}$ was taken equal to $\lambda$ ). The quantity $\lambda_{S} C_{0}$ can be tentatively interpreted as the interaction "volume" for strangeness production events, which may be different 
from the one leading to purely pionic final states.

In practice, the parameters $\lambda, \lambda_{S}, \beta_{S}$ are fitted to the following observables:

$$
\begin{aligned}
& F_{\pi}=\sum_{n=2}^{\infty} f_{0}(n \pi) \\
& \langle n\rangle=F_{\pi}^{-1} \sum_{n=2}^{\infty} n f_{0}(n \pi) \\
& \langle l\rangle=\left(1-F_{\pi}\right)^{-1} \sum_{l=0}^{\infty} l f_{0}(K \bar{K}, l \pi)
\end{aligned}
$$

whose experimental values are the following: ${ }^{17}$ $F_{\pi}=(95.4 \pm 1) \%,\langle n\rangle=5.01 \pm 0.23,\langle l\rangle \approx 1.95$, for annihilations at rest. It should be stressed, however, that the statistical model, described above, reproduces fairly well many more properties of antiproton-proton and antiproton-neutron annihilations, including the multiplicity distributions. We thus want to extend it to $B>0$ annihilations and to analyze its predictions.

In our recent work, ${ }^{8}$ a fit was realized with $\lambda=1.40$, $\lambda_{S} / \lambda=1.80$, and $\beta_{S}=0.22$ [in our present notation, see Eq. (2.2)]. However the parameters can be varied to some extent (in a correlated way) and still give an acceptable fit. Our best fit is obtained with $\lambda=1.40$, $\lambda_{S} / \lambda=1.80$, and $\beta_{S}=0.18$. The sensitivity of the branching ratios to nonstrange and $K \bar{K}$ channels is roughly given in Fig. 1. The sensitivity is slightly larger for branching ratios to channels containing an hyperon.

For $B>0$ annihilations, the generalization of Eqs. (2.1) and (2.2) is provided by the following equations:

$$
\begin{aligned}
& f_{B}(B N, n \pi)=A_{B}\left(\lambda C_{B}\right)^{B+n-1} R_{B+n}\left(\sqrt{s} ; B m_{N}, n m_{\pi}\right), \quad n \geq 2-B, \quad n \geq 0, \\
& f_{B}(B N, K \bar{K}, l \pi)=A_{B} \beta_{S}\left(\lambda_{S} C_{B}\right)^{B+l-1} R_{B+l}\left(\sqrt{s} ; B m_{N}, 2 m_{K}, l m_{\pi}\right), \quad l \geq 0, \\
& f_{B}((B-1) N, Y K, p \pi)=A_{B} \beta_{S}\left(\lambda_{S} C_{B}\right)^{B+p} R_{B+p-1}\left(\sqrt{s} ; B m_{N}, 2 m_{Y}, p m_{\pi}\right), \quad p \geq 0, \\
& f_{B}((B-1) N, \Xi, 2 K, q \pi)=A_{B} \beta_{S}^{2}\left(\lambda_{S} C_{B}\right)^{B+q+1} R_{B+q+2}\left(\sqrt{s} ;(B-1) m_{N}, m_{\Xi}, 2 m_{K}, q m_{\pi}\right), \quad q \geq 0 .
\end{aligned}
$$

The symbol $Y$ denotes a $\Lambda$ or a $\Sigma$ hyperon. Here we have written down isospin averaged formulae. We limit ourselves to the channels described above, but we checked that channels containing $K K K$ or two $Y$ hyperons, when possible (for $B=2,3, \ldots$, e.g.) represent less than $1 \%$. In Eqs. (2.6)-(2.9), we took

$$
C_{B}=[(B+2) / 2]^{2 / 3} C_{0},
$$

keeping on with the idea of Ref. 18 that the interaction volume is proportional to the mass of the initial system. We also retained the idea that $\beta_{s}$ is the hindrance factor for producing strange quarks. Thence, the factor $\beta_{s}^{2}$ appearing in the Eq. (2.9), since this channel requires the creation of two $s \bar{s}$ pairs. Table I shows the predicted rates for $B=1-4$ annihilations, using the same set of parameters as for $B=0$.

The striking result is the enhancement of strangeness production in $B>0$ annihilations compared to $B=0$. Note that the enhancement is basically due to the channels containing $Y K$, while the $K \bar{K}$ channels are depleted. As we explained in Ref. 4, this is a purely kinematical effect. The possibility of $Y K$ lowers the strangeness production threshold energy as compared to $K \bar{K}$. To quantify the strangeness enhancement, we plotted in Fig. 1, the percentage of nonstrange channels,

$$
\eta_{B}=\sum_{n} f_{B}(B N, n \pi),
$$

and also the quantity,

$$
R_{B}=\frac{n(\bar{s})+n(s)}{n(\bar{q})+(n(q)-3 B)},
$$

which represents the abundance of strange quarks relative to nonstrange $(q=u, d)$ quarks. This ratio, in the present case equal to $n(\bar{s}) / n(\bar{q})$, is directly comparable to thermal models for quark production in quark-gluon plasma and also in hadronic matter. For $B=0$ and 1 , one has

$$
R_{0}=\frac{f_{0}(K \bar{K})}{\langle n\rangle+f_{0}(K \bar{K})}
$$

and

$$
R_{1}=\frac{f_{1}(N K \bar{K})+f_{1}(\Lambda K)+f_{1}(\Sigma K)+2 f_{1}(\Xi K K)}{\langle n\rangle+f_{1}(N K \bar{K})},
$$

and similar expressions for $B>1$. In Eqs. (2.12) and (2.13), we use short hand notations: for instance, $f_{0}(K \bar{K})$ stands for the summation of $f_{0}(K \bar{K}, l \pi)$ for all the values of $l$ and $\langle n\rangle$ is the average number of pions in purely pionic channels. Figure 1 shows that the number of nonstrange channels substantially decreases when going from $B=0$ to $B=1$ but tends to stabilize for further increasing values of $B$. On the other hand the strangeness content continues to increase, at a slower pace however, when $B$ increases. This is mainly due to the fact that the average number $\langle n\rangle$ of pions is decreasing. As we said, the great change from $B=0$ to $B=1$ arises from the possibility of production a $\Lambda K$ pair instead of leading to $N K \bar{K}$. For $B>0$, the available energy has to be shared between creating mesons and providing the kinetic energy of the $B$ baryons. This explains why the pion multiplicity regularly decreases with $B$. In particular, while the average number of pions (for any kind of final states) decreases regularly with $B$, the total number $\langle N\rangle$ of particles keeps increasing almost linearly. We also study an extension of this model to in-flight annihilations. At small antiproton 
momentum $p_{\text {lab }}$, the main feature is the increasing of the available c.m. energy. But when $p_{\text {lab }}>2 \mathrm{GeV} / c$, one has to refine the model to cope with two experimental facts: (1) the average number of pions $\langle n\rangle$ increases more slowly than calculated with Eqs. (2.1) and (2.2), which roughly predicts a linear increase with $s$; $(2)$ the proportion of kaonic annihilations increases less rapidly than predicted by Eqs. (2.1) and (2.2). However, the statistical approach should not be rejected. Indeed, the emitted pions are fairly isotropic for a rather large domain of $p_{\text {lab }} \cdot{ }^{19}$ Therefore, we propose to adopt the following generalizations: (a) the interaction "volume" $C_{0}$ should be taken energy dependent as in Ref. 20,

nonstrange yield
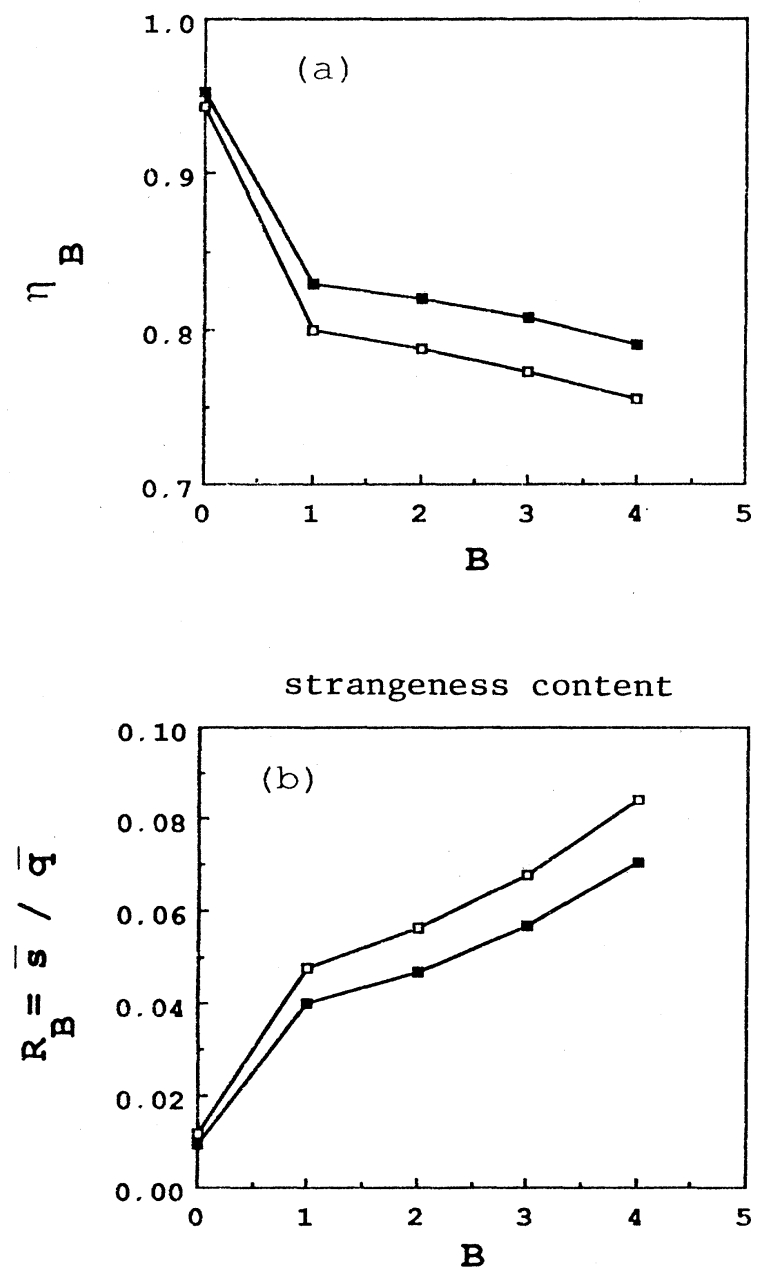

FIG. 1. (a) gives the fraction of nonstrange channels $\eta_{B}$ [containing only pions, and nucleons for $B>0$, Eq. (2.10)] as a function of the baryon number $B$ of the annihilating system. (b) gives the strangeness content $R_{B}$ [measured in terms of number of quarks in the final states, Eq. (2.11)]. In each case, the solid symbols refer to the best fit parameters $\beta_{S}, \lambda$, and $\lambda_{S}$ indicated in the Table I caption. The open symbols correspond to values of the parameters giving a less good but still acceptable fit for $B=0$.

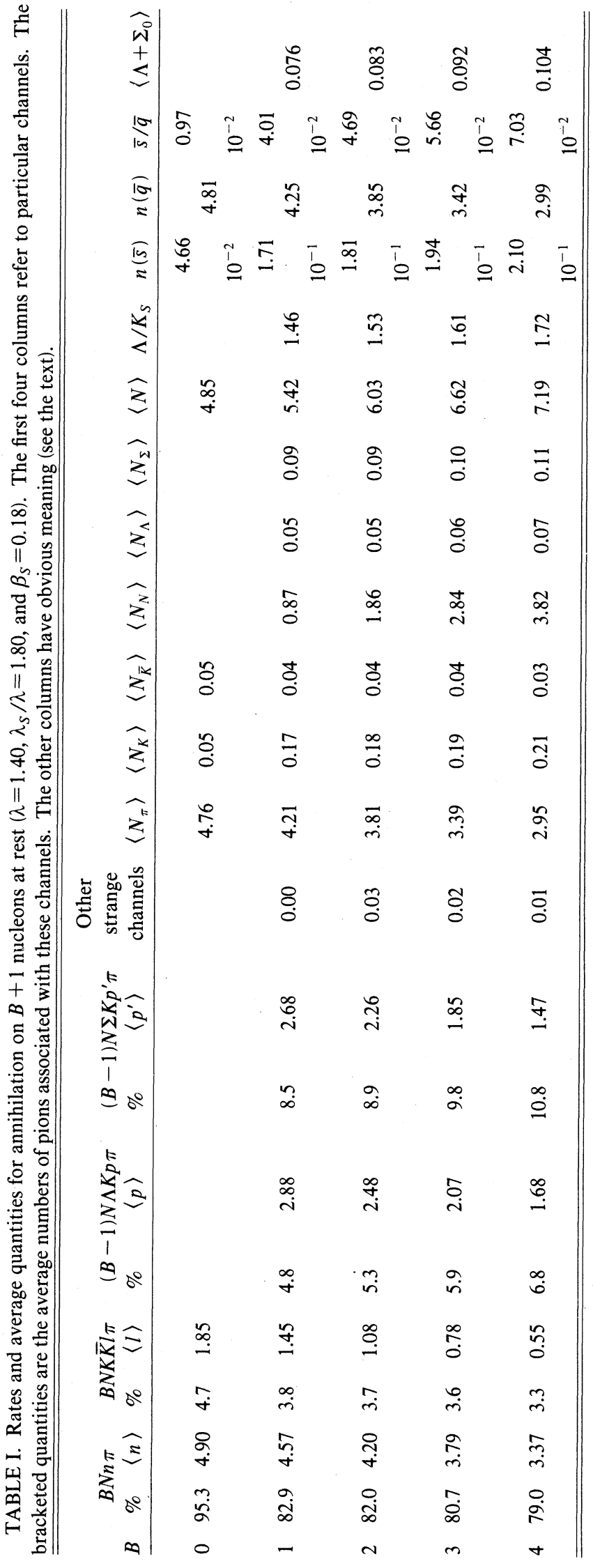




$$
\frac{C_{0}(s)}{C_{0}\left(s_{0}\right)}=h(s)=\frac{s_{0}}{s} \frac{\ln s}{\ln s_{0}},
$$

where $s_{0}=4 M^{2}, M$ being the nucleon mass. This corresponds roughly to a reduction of the interaction volume as $s$ [in units of $\mathrm{GeV}^{2}$ in Eq. (2.14)] increases; (b) the quantity $\rho=\lambda_{S} / \lambda$ is taken to be energy dependent as

$$
\rho(s)=1+\left[\rho\left(s_{0}\right)-1\right] h(s) .
$$

The first modification looks physically plausible, since the interaction time is expected to decrease with the incident energy. The second modification is equally acceptable, since it is reasonable to admit that at high energy a symmetry is progressively restored between the role of mesons of different mass $(\pi, K)$. In any case, the modifications (2.14) and (2.15) provide a good description of the antiproton-proton experimental data up to about $10 \mathrm{GeV} / c$, as it is shown in detail in Refs. 20 and 21.

We give in Fig. 2 the variation of the $K \bar{K}$ production yield and compare it with experiment. ${ }^{22}$ To give an idea of the importance of the modifications (2.14) and (2.15) the predicted yield at $6 \mathrm{GeV} / c$ would be 0.38 if the latter were removed.

The main prediction of this extended model is contained in Fig. 3. As energy increases, for $B=0$, the number of $s$ quarks and the number of $q$ quarks steadily increase. Their ratio is moderately increasing: it is more and more easy to create $K \bar{K}$ pairs. For $B=1$, both numbers are increasing, but their ratio, after rising to a maximum at $p_{\text {lab }}=4 \mathrm{GeV} / c$, slightly decreases. We explain this effect as follows. The pion production is somehow hindered by the possibility of the $\Lambda K$ channel. As the energy increases, the hindrance is less effective because one goes more and more away from the threshold. As Fig. 3 shows, the increase of strangeness production with energy is nevertheless substantial. At $6 \mathrm{GeV} / c$, in $B=1$ an-

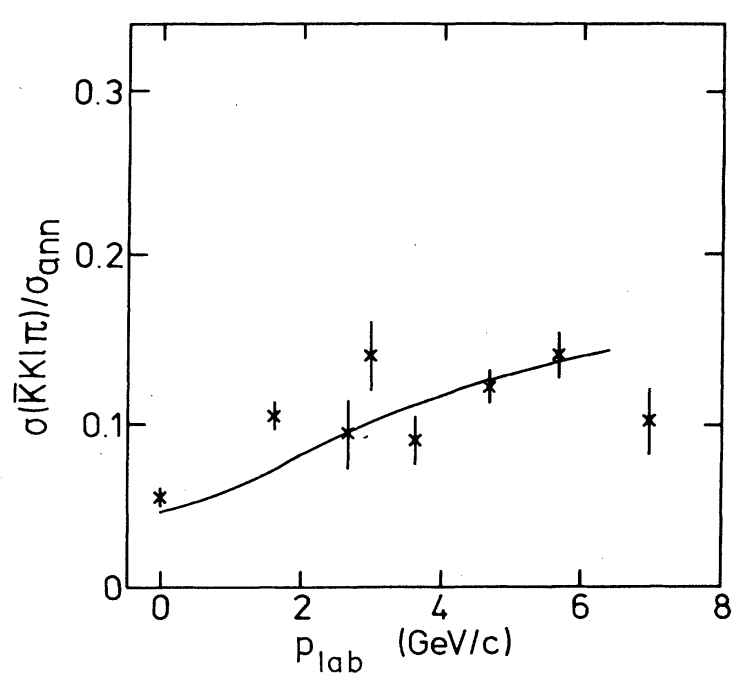

FIG. 2. Comparison of the $K \bar{K} l \pi$ branching ratio as calculated in this paper (curve) with the experimental data of Ref. 22. nihilations, the yield of strange channels reaches $50 \%$ and then keeps increasing very slowly.

We also calculated many quantities in addition to the yield. For instance, in Fig. 4, we show the energy spectrum of various particles. For $B=0$, the pions appear thermal-like with a temperature of $T=110 \mathrm{MeV}$. This temperature is lowered a little bit when one goes from $B=0$ to $B=1$. This is so because the available energy should be shared to more particles. The spectrum of the nucleons in $B=1$ strikingly shows the influence of the microcanonical approach since the change of slope corresponds to nucleons at the edge of the available phase space compatible with the energy-momentum conservation law. The difference between nucleon and hyperon shapes comes from the fact that in the latter case, one is almost at the edge of phase space even for slow moving hyperons. This comes from the part of the available energy which is transformed in mass. This situation also applies to the kaon spectrum for $B=0$. The difference of temperatures of pions and slow ("canonical") nucleons can be explained by the fact that our model is not a standard thermal model as far as pions are concerned. Indeed, the model neither assumes a constant number of
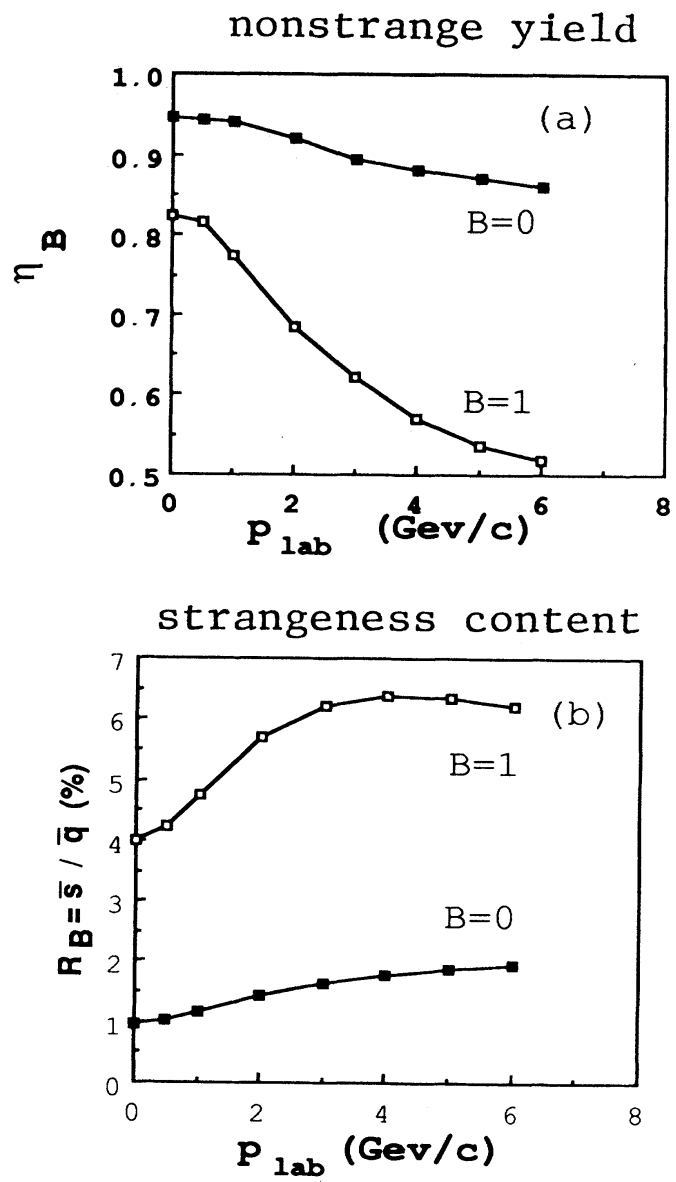

FIG. 3. Evolution of nonstrange yield (upper part) and of the strangeness content (lower part), both in $B=0$ (solid symbols) and in $B=1$ annihilations (open symbols), as a function of the incident momentum $p_{\text {lab }}$ of the antiproton. 


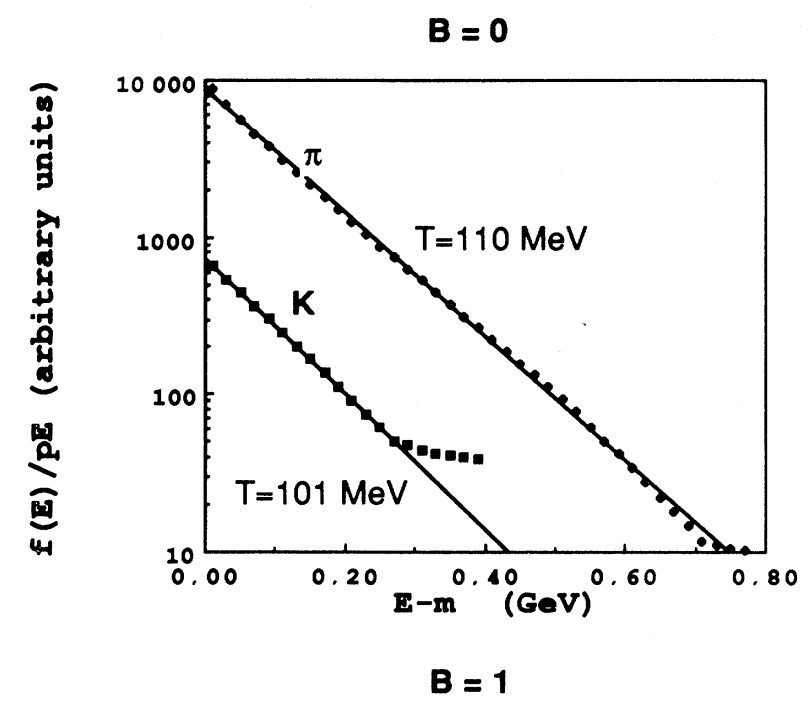

$$
\eta=\sum_{B=0}^{A-1} P_{B} r_{B} D_{B}
$$

where $P_{B}$ denotes the probability that the annihilation occurs on $(B+1)$ nucleons, $r_{B}$ is the branching ratio for the particular class of events in that case, and $D_{B}$ represents the distortion due to the subsequent cascades. In most cases, it simply is an average absorption coefficient.

The most difficult task is, of course, the computation of $D_{B}$ which requires an elaborate cascade calculation. We here concentrate on the probabilities $P_{B}$, for which one may build a simple geometrical model for annihilation in flight. We assume that the incoming antiproton forms with a nucleon a $B=0$ fireball which can decay with some lifetime $\tau_{0}$, but which can form a $B=1$ fireball, prior to that, if it encounters another nucleon. We call $\sigma_{1}$ the associated cross section. The $B=1$ fireball is provided with a lifetime $\tau_{1}$ and a cross section $\sigma_{2}$ for forming a $B=2$ fireball, and so on. To simplify the calculations, we rely on the Glauber picture of a forward motion. The model may be put in the form of rate equations

$$
\begin{aligned}
& \frac{d Q_{0}(z)}{d z}=-\sigma_{0} \rho(z) Q_{0}(z), \\
& \frac{d R_{0}(z)}{d z}=\sigma_{0} \rho(z) Q_{0}(z)-\left(\frac{1}{\gamma_{0} v_{0} \tau_{0}}+\sigma_{1} \rho(z)\right) R_{0}(z),
\end{aligned}
$$

and

$$
\frac{d R_{i}(z)}{d z}=\sigma_{i} \rho(z) R_{i-1}(z)-\left(\frac{1}{\gamma_{i} v_{i} \tau_{i}}+\sigma_{i+1} \rho(z)\right) R_{i}(z),
$$

FIG. 4. Energy spectrum $f(E)$ for various emitted particles, both for $B=0$ and for $B=1$ annihilations. The spectrum is divided by the product of the momentum by the total energy. In this representation a thermal spectrum appears as a straight line. Temperatures are tentatively extracted and displayed.

pions nor a constant chemical potential.

The change of slope at the extreme tail of the spectra, especially for $B=0$, is due to the contribution of the three-body decay (the two-body is not included in the figure), while the main part is due mainly to $n$-body decays, with $n \geq 4$.

Other quantities, like, for instance, the shape of the multiplicity distributions, show interesting variations when $B$ is changed, but one can say, in conclusion, that none shows a qualitatively important change as the strangeness yield.

\section{NUCLEAR EFFECTS}

The important issue is to detect the existence of $B>0$ annihilations in antiproton annihilation on a nucleus. Let us suppose we focus our attention on a special kind of event. To fix the ideas, this may correspond to the detection of a $K^{+}$. In all generality, the yield per annihilation for such kinds of events, on a target of mass number $A$, can be written as

for $i=1,2, \ldots, n$. Here, $z$ is the coordinate along the trajectory of the antiproton or its subsequent fireball inside the nucleus; $v_{i}$ is the velocity of the $B=i$ fireball and $\gamma_{i}$ is the associated Lorentz factor. The quantity $\sigma_{0}$ is the $\bar{p} N$ annihilation cross section. At $z=-\infty$, the probability $Q_{0}$ of having an antiproton is equal to unity and the probability $R_{i}$ of having a $B=i$ fireball is vanishing. The connection between the quantities $P_{B}$ of Eq. (3.1) and the functions $R_{B}(z)$ is given by

$$
P_{B}=\lim _{z \rightarrow \infty} \frac{\int_{-\infty}^{z}\left(\gamma_{B} v_{B} \tau_{B}\right)^{-1} R_{B}(y) d y}{1-Q_{0}(z)},
$$

The use of the Glauber picture is more or less justified by the fact that the latter provides a surprisingly good description of the antiproton reaction cross sections. ${ }^{23}$ The predictions of this model should not be taken too seriously: it is aimed at providing rough estimates of the $P_{B}$ 's only. In particular, only reasonable guesses of the quantities $\tau_{i}$ and $\sigma_{i}(i>1)$ are available. We give in Fig. 5 the decay rates of the fireball of baryon number $i$ [i.e., the term containing the $\tau_{i}$ in Eqs. (3.2b) and (3.2c)] in a typical example and with typical parameters $\sigma_{i}=\sigma_{0}$ $(i \geq 1)$ and $\tau_{i}=1 \mathrm{fm} / \mathrm{c}(i \geq 0)$. For simplicity, we took the 


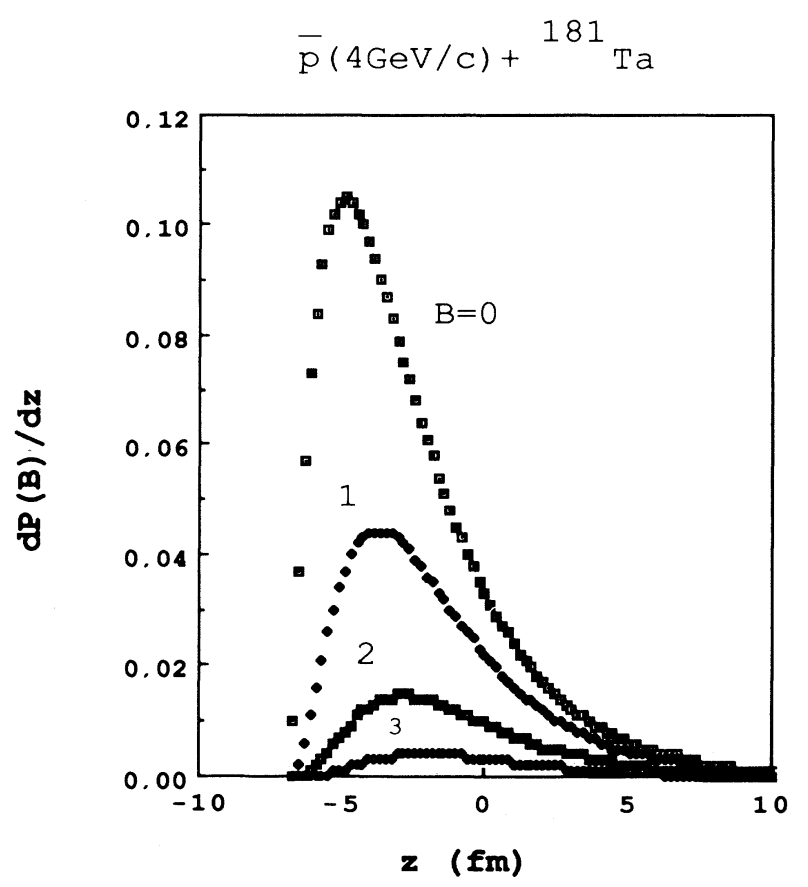

FIG. 5. Evolution as a function of the distance $z$ of the decay probability for the fireballs of baryon number $B=0$ to 3 , respectively, in the case of central antiproton annihilation on Ta nucleus. The target nucleus is centered at $z=0$.

same $p_{\text {lab }}$ dependence for all cross sections. The figure reveals that most of the annihilations happen rather close to the surface. There is, however, a slight shift from the average location of annihilations when $B$ is going up. In Fig. 6 we show the results of the same model when different, but still reasonable assumptions are made for the quantities $\sigma_{i}$ and $\tau_{i}$. In one case, we assumed that $\sigma_{i}$ increases when $i$ increases, with a geometrical law

$$
\sigma_{B}=\left(\frac{(B+1)^{1 / 3}+1}{2}\right)^{2} \sigma_{0} .
$$

In the other case, the lifetime is assumed to decrease with the mass number as

$$
\tau_{B}=\left(\frac{2}{B+2}\right) \tau_{0},
$$

and $\tau_{0}=1 \mathrm{fm} / c$. As one can see, these modifications do not influence the results dramatically. Therefore, we can safely conclude that on the basis of reasonable geometric assumptions, the annihilation rate for $B>0$ should be of

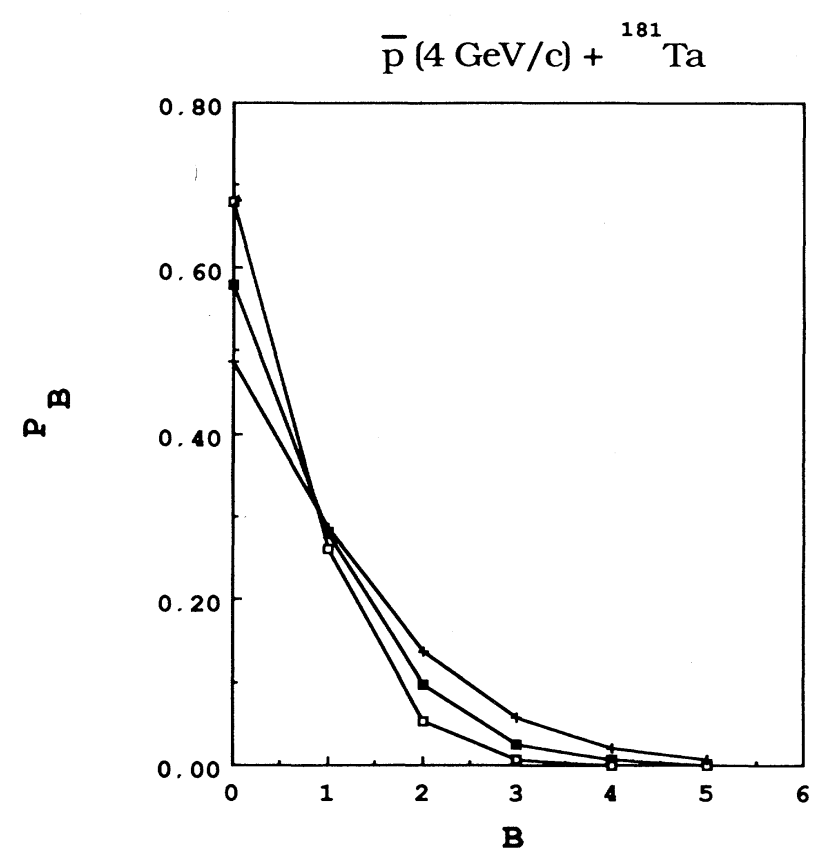

FIG. 6. Distribution of the quantities $P_{B}$ [Eq. (3.1)] for central annihilation of antiprotons on Ta nuclei. The solid squares correspond to $\sigma_{i}=\sigma_{0}$ and $\tau_{i}=1 \mathrm{fm} / c$. The open squares are obtained with Eq. (3.5) and the crosses with Eq. (3.4) (see the text for details).

the order of $10 \%$ in actual nuclei. However, it is not likely to have very large values of $B$. The most important parameter to favor large $B$ is the lifetime $\tau$. But, we have increased the lifetime $\tau_{0}$ up to $5 \mathrm{fm} / c$. We observed a maximum of $P_{B}$ at $B=2$ only, although the distribution is much broader. In Fig. 7, we display the population probability for the baryon number $B$ of the fireball in the limiting case of $\tau_{i} \rightarrow \infty$ [keeping Eq. (3.4)]. In this case, the fireballs grow up to a mass which roughly corresponds to the number of nucleons intercepted by the cross section $\sigma_{0}$ at that energy. This number turns out to be around 6 .

The short-range correlations between nucleons limits the piling up of the fireballs. This can be accounted for in Eqs. (3.2b) and (3.2c) by multiplying $\rho(z)$ in all terms involving the quantities $R_{i}$ by the function $g(z)$ which represents the probability of having two nucleons at a distance $z$ from each other. We checked that with reasonable forms ${ }^{24}$ of $g(z)$, this amounts to introducing an effective cross section about $60 \%$ of the original $\sigma_{i}$ 's.

Finally, we give in Table II the values of the probabilities of having various $B$ annihilations on the nuclei ${ }^{3} \mathrm{He}$

TABLE II. Probability $P_{B}$ of having a $B$ annihilation on ${ }^{3} \mathrm{He}$ and ${ }^{4} \mathrm{He}$ targets.

\begin{tabular}{lccccccc}
\hline \hline & \multicolumn{3}{c}{${ }^{3} \mathrm{He}$} & ${ }^{3} \mathrm{He}$ & $P_{3}$ \\
\hline$p_{\text {lab }}(\mathrm{GeV} / c)$ & $P_{0}$ & $P_{1}$ & $P_{2}$ & $P_{0}$ & $P_{1}$ & $P_{2}$ & $P_{3}$ \\
0.4 & 0.900 & 0.088 & 0.011 & 0.885 & 0.099 & 0.015 & 0.00021 \\
0.6 & 0.886 & 0.100 & 0.014 & 0.866 & 0.113 & 0.019 & 0.00043 \\
1 & 0.873 & 0.110 & 0.016 & 0.846 & 0.128 & 0.024 & 0.00080 \\
2 & 0.868 & 0.114 & 0.016 & 0.836 & 0.136 & 0.025 & 0.0014 \\
4 & 0.882 & 0.105 & 0.013 & 0.849 & 0.128 & 0.020 & 0.0014 \\
\hline \hline
\end{tabular}




\section{$\overline{\mathrm{p}}(4 \mathrm{GeV} / \mathrm{c})+{ }^{181} \mathrm{Ta}$}

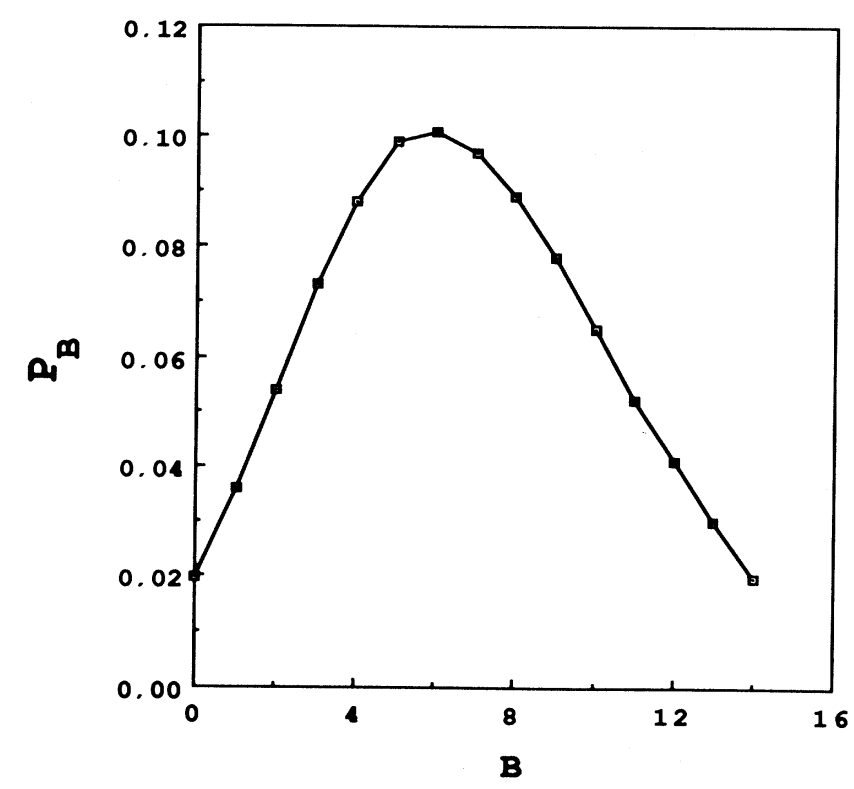

FIG. 7. Probability distribution of the baryon number $B$ of the fireball [quantities $R_{B}$ in Eq. (3.2)] at the outcome of the target in the limit of $\tau_{B} \rightarrow \infty$ (see the text for details).

and ${ }^{4} \mathrm{He}$ (which presents some cosmological interest ${ }^{25}$ ). In this particular case, we have summed over all possible impact parameters, using standard assumptions and Gaussian density distributions with parameters taken from Ref. 26. These values will be used in Sec. IV.

\section{TWO-BODY EXIT CHANNELS}

The most convincing fact in favor of $B>0$ annihilations is the observation of two-body decays (with clear kinematical signature) like the following one: $\bar{p} d \rightarrow p \pi^{-}$. We want here to present the predictions of our model for this kind of process. Once again, we checked that the model works fairly well for $B=0$ (see Table III). This gives us some confidence in extending the model for $B>0$. Let us detail a little bit the results.

$$
\text { A. } B=1
$$

The branching ratio for $\bar{p} d \rightarrow p \pi^{-}$in $B=1$ [quantity $r_{1}$ in Eq. (3.1)] is predicted to be $4.7 \times 10^{-4}$ (relative to Table III, there is an isospin factor $\frac{2}{3}$ ). A comparison of formula (3.1) with the experimental rate (Ref. 28), $(28 \pm 3) \times 10^{-6}$, yields a probability $P_{1}=(6 \pm 0.6) \%$ of having $B=1$ annihilations (in the case of kinematically defined two-body decays, the distortion factors $D_{B}$ are equal to one). Taking this value of $P_{1}$, we can make predictions for $\bar{p} d \rightarrow \Lambda^{0} K^{0}$ and $\bar{p} d \rightarrow \Sigma^{-} K^{+}$yields, i.e., about $6 \times 10^{-6}$ and about $12 \times 10^{-6}$ per annihilation, respectively.

One may look at the predictive power of our model from another point of view. Indeed, the ratio of the following yields

$$
\rho_{1}=\frac{\eta\left(\bar{p} d \rightarrow \Sigma^{-} K^{+}\right)}{\eta\left(\bar{p} d \rightarrow p \pi^{-}\right)}
$$

is independent of the quantity $P_{1}$ [Eq. (3.1)], and tests the statistical part of our model (Sec. II). Indeed, $\rho_{1}$ writes

$$
\rho_{1}=\beta_{S} \frac{\lambda_{S}}{\lambda} \frac{p_{\Sigma}^{*}}{p_{p}^{*}}
$$

where $p^{*}$ denotes the c.m. momentum in the final state, and is directly connected to the hindrance factor $\beta_{s}$. Similarly, the ratio

$$
\rho_{2}=\frac{\eta\left(p \bar{p} \rightarrow K^{+} K^{-}\right)}{\eta\left(p \bar{p} \rightarrow \pi^{+} \pi^{-}\right)}=\frac{3}{5} \beta_{S} \frac{\lambda_{S}}{\lambda} \frac{p_{K}^{*}}{p_{\pi}^{*}}
$$

is proportional to the hindrance factor. The crucial step in Sec. II is to assume that the hindrance factor is the same for $B=0$ and $B=1$. Therefore, the quantities $\rho_{1}$ and $\rho_{2}$ are really testing the model. In particular, the equivalence of the hindrance factors implies

$$
\frac{\rho_{1}}{\rho_{2}}=\frac{5}{3} \frac{p_{\Sigma}^{*}}{p_{p}^{*}} \times \frac{p_{\pi}^{*}}{p_{K}^{*}}
$$

The value of the right-hand side for annihilations at rest is $\sim 1.7$. The experimental value of $\rho_{2}$ is known to be $\sim 0.33$. Therefore, if our assumption is correct, $\rho_{1}$ should be of $\sim 0.28$. Experimentally $\rho_{1}$ is not known. If the $\bar{p} d \rightarrow p \pi^{-}$yield is $(28 \pm 3) \times 10^{-6}$, the $\bar{p} d \rightarrow \Sigma^{-} K^{+}$one should be of the order ${ }^{29}$ of $(15 \pm 1.5) \times 10^{-6}$. This is to be

\begin{tabular}{|c|c|c|c|c|c|c|c|c|c|c|}
\hline & & & & \multicolumn{3}{|c|}{$B=0$} & \multicolumn{3}{|c|}{$B=1$} & \multirow{2}{*}{$\begin{array}{c}B=2 \\
N N\end{array}$} \\
\hline & & $\pi \pi$ & $K \bar{K}$ & $\bar{p} p \rightarrow \pi^{+} \pi^{-}$ & $\bar{p} p \rightarrow K^{+} K^{-}$ & $\bar{p} p \rightarrow K^{0} \bar{K}^{0}$ & $N \pi$ & $\Lambda K$ & $\Sigma K$ & \\
\hline \multirow[t]{4}{*}{ Theory } & & 0.51 & 0.14 & 0.42 & 0.07 & 0.07 & 7.06 & 1.03 & 3.00 & 1.06 \\
\hline & & $\times 10^{-2}$ & $\times 10^{-2}$ & $\times 10^{-2}$ & $\times 10^{-2}$ & $\times 10^{-2}$ & $\times 10^{-4}$ & $\times 10^{-4}$ & $\times 10^{-4}$ & $\times 10^{-4}$ \\
\hline & liquid & & & $(0.37 \pm 0.02)$ & $(0.11 \pm 0.01)$ & $(0.072 \pm 0.010)$ & & & & \\
\hline & & & & $\times 10^{-2}$ & $\times 10^{-2}$ & $\times 10^{-2}$ & & & & \\
\hline \multirow{4}{*}{ Expt. } & & & & $(0.32 \pm 0.03)$ & $(0.096 \pm 0.008)$ & $(0.081 \pm 0.005)$ & & & & \\
\hline & gas & & & $\times 10^{-2}$ & $\times 10^{-2}$ & $\times 10^{-2}$ & & & & \\
\hline & & & & & & $(0.051 \pm 0.012)$ & & & & \\
\hline & & & & & & $\times 10^{-2}$ & & & & \\
\hline
\end{tabular}
compared with a $90 \%$ confidence upper limit of about $8 \times 10^{-6}$, according to Ref. 27. An improvement of this

TABLE III. Calculated and measured branching ratios for several two-body final states in various $B$ annihilations. The liquid hydrogen data are taken from the compilation of Ref. 17. The gas data are from Ref. 27. 
measurement is clearly needed to confirm or infirm our approach.

Let us note that the $\bar{p} d \rightarrow p \pi^{-}$yield has recently been measured by another group ${ }^{30}$ which finds a rate of $(14 \pm 7) \times 10^{-6}$, somewhat lower than the value of Ref. 28.

$$
\text { B. } \boldsymbol{B}=2
$$

This case is particularly interesting since it corresponds to the emission of two nucleons without any meson: the so-called Pontecorvo reactions. ${ }^{1}$ There are four of them:

$$
\begin{aligned}
& \bar{p}^{3} \mathrm{He} \rightarrow p n, \\
& \bar{p} t \rightarrow n n, \\
& \bar{n} t \rightarrow p n, \\
& \bar{n}^{3} \mathrm{He} \rightarrow p p .
\end{aligned}
$$

The most interesting ones are the first one and, perhaps even more, the last one because the final state contains two charged particles. Combining the results of Tables II and III, we can make a prediction of the yield for this particular reaction. The latter turns out to be of the order of $10^{-6}$, which seems to be just sufficient to make a measurement possible with the present beam and detection possibilities. Our prediction is to be contrasted with the result of Ref. 31 , where the authors give an estimate of $10^{-7}-10^{-8}$, but using a very specific six-quark intermediate state.

\section{ANTILAMBDA ANNIHILATION}

In the near future, experiments with antilambdas will be possible with good statistics. For this reason, we present here the results of our model for this case. The corresponding expressions are then (for $B=0$ )

$$
\begin{aligned}
& f_{0}(K, n \pi)=A_{0}\left(\lambda C_{0}\right)^{n} R_{n+1}\left(\sqrt{s} ; m_{K}, n m_{\pi}\right), \quad n \geq 1, \\
& f_{0}(K K \bar{K}, l \pi)=A_{0}\left(\lambda_{S} C_{0}\right)^{l+2} R_{l+1}\left(\sqrt{s} ; 3 m_{K}, l m_{\pi}\right), \quad l \geq 0,
\end{aligned}
$$

and (for $B=1$ )

$$
\begin{aligned}
& f_{1}(N, K, n \pi)=A_{1}\left(\lambda C_{1}\right)^{n+1} R_{n+2}\left(\sqrt{s} ; m_{N}, m_{K}, n m_{\pi}\right), \quad n \geq 0, \\
& f_{1}(N, K K \bar{K}, l \pi)=A_{1} \beta_{S}\left(\lambda_{S} C_{1}\right)^{l+3} R_{l+4}\left(\sqrt{s} ; m_{N}, 3 m_{K}, l m_{\pi}\right), \quad l \geq 0, \\
& f_{1}(Y, K K, p \pi)=A_{1} \beta_{S}\left(\lambda_{S} C_{1}\right)^{p+2} R_{p+3}\left(\sqrt{s} ; m_{Y}, 2 m_{K}, p m_{\pi}\right), \quad p \geq 0,
\end{aligned}
$$

for the most important channels. We used the same values of the coefficients as before, as well as the same $s$ dependence.

The results of the antilambda annihilation at rest are presented in Table IV and compared to the antinucleon annihilation case. One can realize that the rates for the corresponding channels in the two cases (both for $B=0$ and $B=1)$ are very similar. The multiplicities are slight-

\begin{tabular}{|c|c|c|c|c|c|c|c|c|}
\hline & \multicolumn{4}{|c|}{$B=0$} & \multicolumn{4}{|c|}{$B=1$} \\
\hline & \multicolumn{2}{|c|}{$\bar{N} N$} & \multicolumn{2}{|c|}{$\bar{\Lambda} N$} & \multicolumn{2}{|c|}{$\bar{N} N N$} & \multicolumn{2}{|c|}{$\bar{\Lambda} N N$} \\
\hline & $n \pi$ & 95.34 & $K n \pi$ & 96.47 & $N n \pi$ & 82.94 & $N K n \pi$ & 86.76 \\
\hline & $\langle n\rangle$ & 4.90 & $\langle n\rangle$ & 3.57 & $\langle n\rangle$ & 4.57 & $\langle n\rangle$ & 3.21 \\
\hline & $K \bar{K} l \pi$ & 4.66 & $K K \bar{K} l \pi$ & 3.53 & $N K \bar{K} l \pi$ & 3.76 & $N K K \bar{K} l \pi$ & 2.37 \\
\hline & $\langle l\rangle$ & 1.85 & $\langle l\rangle$ & 0.54 & $\langle l\rangle$ & 1.45 & $\langle l\rangle$ & 0.32 \\
\hline & & & & & $\Lambda K p \pi$ & 4.79 & $\Lambda K K p \pi$ & 3.93 \\
\hline & & & & & $\langle p\rangle$ & 2.69 & $\langle p\rangle$ & 1.45 \\
\hline & & & & & $\Sigma K p^{\prime} \pi$ & 8.49 & $\Sigma K K p^{\prime} \pi$ & 6.94 \\
\hline & & & & & $\left\langle p^{\prime}\right\rangle$ & 2.68 & $\left\langle p^{\prime}\right\rangle$ & 1.25 \\
\hline$\left\langle N_{\pi}\right\rangle$ & & 4.76 & & 3.46 & & 4.21 & & 2.93 \\
\hline$\left\langle N_{K}^{\prime}\right\rangle$ & & 0.047 & & 1.035 & & 0.17 & & 1.14 \\
\hline$\left\langle N_{\bar{K}}\right\rangle$ & & 0.047 & & 0.035 & & 0.038 & & 0.024 \\
\hline$\left\langle N_{N}\right\rangle$ & & & & & & 0.867 & & 0.891 \\
\hline$\left\langle N_{\Lambda}\right\rangle$ & & & & & & 0.048 & & 0.039 \\
\hline$\left\langle N_{\Sigma}\right\rangle$ & & & & & & 0.085 & & 0.069 \\
\hline$\langle N\rangle$ & & 4.85 & & 4.53 & & 5.42 & & 5.09 \\
\hline
\end{tabular}
ly smaller in the antilambda case compared to the antinucleon case. This is understandable in view of the fact that strangeness conservation imposes the presence of a $K$ meson in the final state, which requires more energy than the lambda-nucleon mass difference.

The $B=1$ antilambda annihilation leads also to the

TABLE IV. Upper part: branching ratios in \% for several types of final states and associated average pion multiplicities in $B=0$ and $B=1$ annihilations of antinucleons and of antilambdas. Lower part: average multiplicities for various particles. 
TABLE V. Comparison of the calculated $\bar{N} N N \rightarrow N \pi$ and $\bar{\Lambda} N N \rightarrow N K$ branching ratios as a function of the incident momentum.

\begin{tabular}{llc}
\hline \hline$p_{\text {lab }}$ & $\bar{N} N N \rightarrow N \pi$ & $\bar{\Lambda} N N \rightarrow N K$ \\
\hline 0 & $7.06 \times 10^{-4}$ & $1.46 \times 10^{-3}$ \\
0.5 & $4.79 \times 10^{-4}$ & $1.07 \times 10^{-3}$ \\
1 & $1.99 \times 10^{-4}$ & $5.08 \times 10^{-4}$ \\
2 & $2.88 \times 10^{-5}$ & $7.81 \times 10^{-5}$ \\
3 & $5.28 \times 10^{-6}$ & $1.23 \times 10^{-5}$ \\
4 & $1.34 \times 10^{-6}$ & $2.86 \times 10^{-6}$ \\
5 & $4.28 \times 10^{-7}$ & $8.15 \times 10^{-7}$ \\
6 & $1.64 \times 10^{-7}$ & $3.10 \times 10^{-7}$ \\
\hline
\end{tabular}

possibility of remarkable two-body channels. In particular, the following reaction $\bar{\Lambda} d \rightarrow K^{0} p$ is allowed. We give in Table $\mathrm{V}$ the relative rates for $B=1$ annihilation in flight leading to two particles in the final state, both for antilambda and for antiproton. The branching ratios vary in a fairly similar way as a function of the incident momentum. Taking into account isospin weights, we predict

$$
\frac{\eta\left(\bar{\Lambda} d \rightarrow K^{0} p\right)}{\eta\left(\bar{p} d \rightarrow \pi^{-} p\right)} \approx 1.5
$$

\section{DISCUSSION, CONCLUSION}

In this work, we proposed a picture of the annihilation process, which can be characterized by the following features:

(1) The final state probabilities proceed from a statistical picture. The underlying assumption is that the annihilation process is a rather complicated process, which involves a strong rearrangement of the parton structure. Therefore it is very much alike the compound nucleus formation for the nucleons.

(2) The annihilation on several nucleons occurs whenever possible. Again, this assumption derives from the observation that the fundamental process is the quarkantiquark annihilation.

(3) The statistical picture applies to all annihilations, including those involving more than one nucleon.

(4) Strangeness production is hindered. Furthermore, it is assumed that the hindrance is the same for all kinds of annihilations, irrespective of the number of nucleons involved.

This approach works very well for $B=0$ annihilations. This statement pertains to points (1) and (4) above. We have a very good reproduction of the branching ratios for many final states, of the pion multilicity distributions, of the pion spectrum, of the strangeness production yield, etc. This is obtained with the help of only three free parameters (for annihilation at rest). This part of our approach seems very successful. We think that such a success is not accidental and that the model should cover the correct physical picture. We have to admit however that points (2) and (3) have not yet been tested up to now, even though there are experimental indications in favor of the annihilation on several nucleons. ${ }^{32,33}$ The very observa- tion of the $\bar{p} d \rightarrow p \pi^{-}$reaction ${ }^{28,32}$ strongly suggests that the annihilation process involves two nucleons at the same time. It should not be considered as a proof for the time being, since it can also be pictured as due to the reabsorption of an off-shell pion by the second nucleon. ${ }^{34}$ However, it is not sure that the two approaches exclude each other and that they are not merely two descriptions of the same physical reality using different words and different degrees of complexity. Analyses of various strangeness yields seem to call also for the need $B>0$ annihilations as it is shown in Ref. 35.

An interesting question is to know whether our approach bears some relationship with the current dynamical approaches using the quark structure. To summarize very briefly the situation with the latter, ${ }^{14,36}$ one can say that there are two basic philosophies. In one case, it is assumed that the quark-line diagrams should be ordered with increasing number of annihilation and/or creation quark-antiquark vertex. In the other one, on the contrary, they should be ordered with decreasing character of planarity. Obviously, our statistical model is more closely related to the so-called annihilation diagrams, which are sketched in Fig. 8(a). More precisely, our approach consists in retaining this class of diagrams and in identifying the intermediate state [dotted line in Fig. 8(a)] as a heavy meson which decays statistically. This connection assumed between meson decays and annihilation process should reflect in a similar mean multiplicity of final products. We show in Fig. 9(a), as a function of the

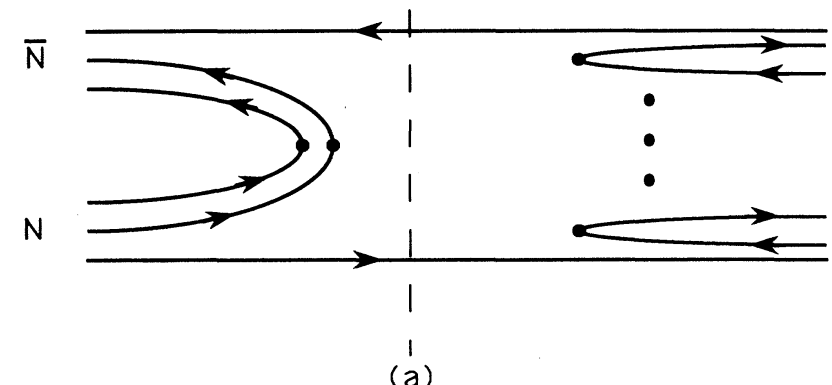

(a)

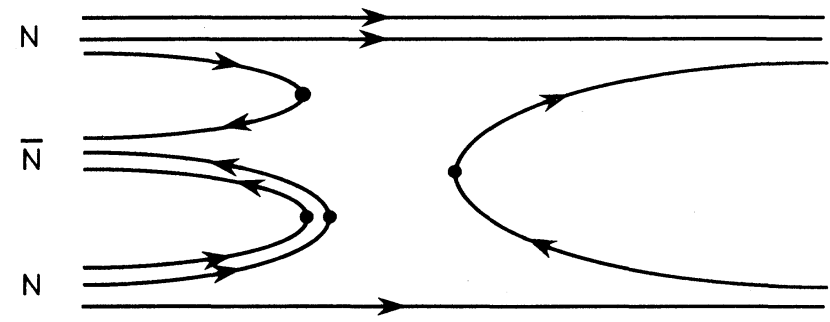

(b)

FIG. 8. (a) "Annihilation" diagram for $B=0$ annihilation; (b) possible "annihilation" diagram for $B=1$. See the text for details. 
mass, the mean multiplicity in the pionic modes of antiproton-proton annihilation at rest and in the decay of the nonstrange mesons which have dominant pionic channels. It is seen that the linear dependence connecting the pion to the antinuclon-nucleon system goes through the meson points in the mass domain around

(a) nonstrange mesons

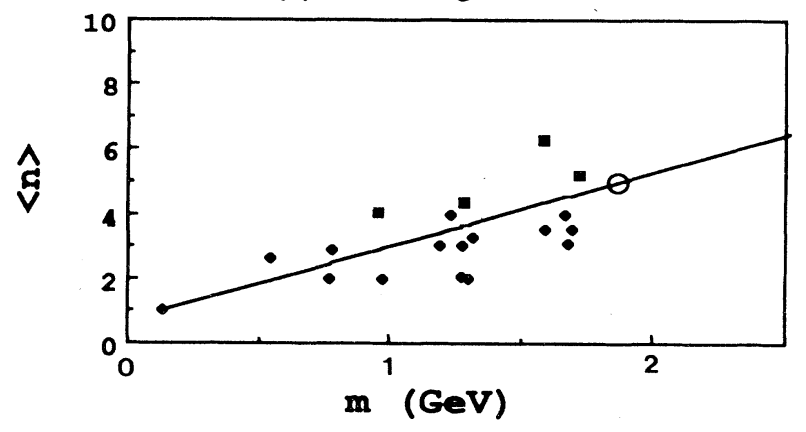

(b) baryonic resonances

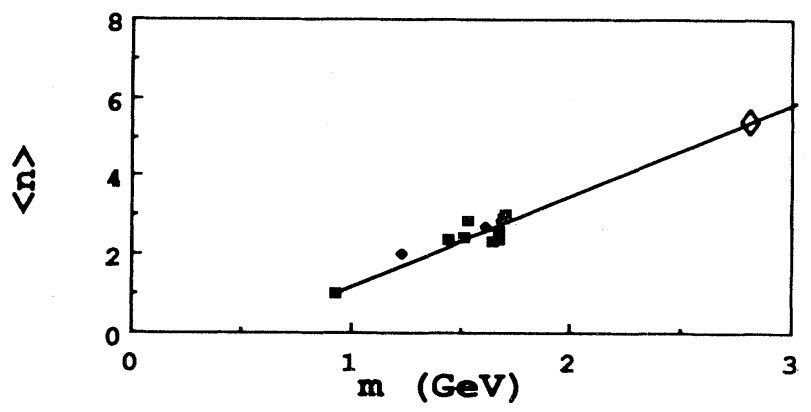

(c) strange mesons

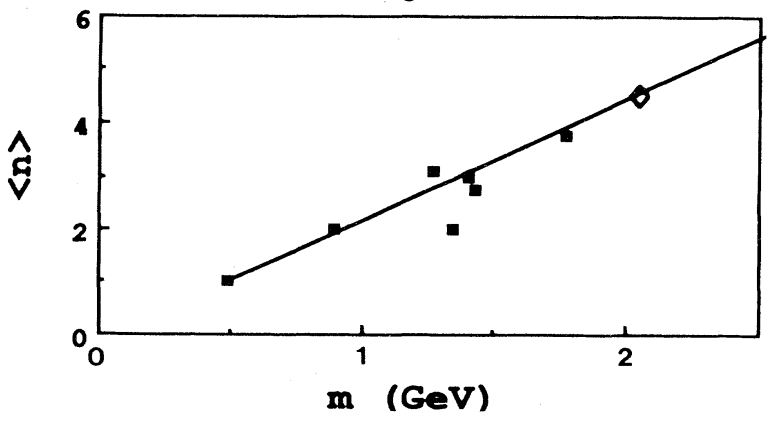

FIG. 9. Multiplicity of stable products $(\pi, K, N$, and $\gamma$ from $\eta$ ) in the decay of various systems: (a) $S=0$ mesons (diamonds and squares, the latter corresponding to the decays involving $\eta$ 's) and antiproton-proton at rest (open circle). (b) $S=0$ baryons (solid diamonds and squares) and $(\bar{N} N N)$ system at rest (open diamond). (c) $S=-1$ mesons (squares) and antilambdanucleon system at rest (open diamond). The open diamonds are our theoretical results while all the other points are experimental ones, taken from Ref. 37 or from Ref. 17 for the open circle in part (a). about $1 \mathrm{GeV}$; beyond about $1.3 \mathrm{GeV}$, the identified resonances tend to cluster below the line, the result of a probable bias favoring the identification of states with low final mean multiplicities. Some states with the $\eta$ meson as a main decay product are known [squares in Fig. 9(a)] and it is obvious that channeling through $\eta$ makes the system more easily identifiable than for a set of more loosely correlated particles.

Once the picture of a mesonlike intermediate state is accepted, some other features of our approach arise naturally. First, strangeness production requires a $s \bar{s}$ creation vertex, whose importance is reduced in comparison with a $q \bar{q}$ vertex because of the mass of the strange quarks. Second, if two nucleons are close enough, it is reasonable to admit that an antiquark in the antinucleon annihilates with a quark in one nucleon bag while another antiquark annihilates with a quark of the other nucleon bag, which corresponds to an annihilation on two nucleons. One possible graph for this process is depicted in Fig. 8(b). Finally, it is natural within this scheme to assume that strangeness production is reduced in the same manner in $B=0$ and $B>0$ annihilations. Let us notice, however, that within this simpleminded quark picture, $B$ should be limited to 0,1 , and 2 .

A test on final multiplicity similar to the one for $B=0$, can be made with the observed nucleon resonances and our prediction for $B=1$ annihilation. As Fig. 9(b) shows, the decay multiplicities of the resonances of $N^{*}$ and $\Delta^{*}$ type cluster around the line joining the nucleon to the $B=1$ system. It is also instructive to consider the strange systems with $B=0$. We have plotted in Fig. 9(c) the experimental figures for the mean product multiplicity of the strange mesons and our prediction for antilambda-nucleon annihilation at rest. The connection works out amazingly well here too.

One of the surprising results of our analysis is that it gives a reasonable yield for the rare channels, i.e., in a sector where a statistical approach is sometimes expected to fail. This definitely applies in $B=0$ annihilations. In that respect, it is interesting to mention that the double production of a $K \bar{K}$ pair seems to be predicted at the right order of magnitude by our model, which includes $\beta_{S}^{2}$ as factor in that case. At $3.66 \mathrm{GeV} / c$ the measured cross section for $\bar{p} p \rightarrow 2 K 2 \bar{K} j \pi(j \geq 0)$ is $\left(3.1_{-2.3}^{+4}\right) 10^{-2}$ $\mathrm{mb}$. We find a fraction of $9 \times 10^{-4}$ which, multiplying a total annihilation cross section of $\approx 30 \mathrm{mb}$ gives a predicted cross section of $\approx 2.7 \times 10^{-2} \mathrm{mb}$. But the agreement between model and experiment can also apply to $B=1$ (see Sec. IV), provided a reasonable frequency of $B=1$ events is assumed in the $\bar{p} d$ system.

The most important issue at present is to know whether $B=1$ annihilations really occur in antiproton (or antibaryon in general) annihilation on atomic nuclei. As we said in the Introduction and in Sec. II, the best indicator seems to be the strangeness production yield. This of course relies on our assumption for a hindrance factor $\beta_{S}$ independent of $B$. As we explained in Sec. IV, this assumption can be tested on the ratio $\rho_{1} / \rho_{2}$, which is independent of the frequency for $B=1$ annihilations. Therefore, accurate measurements of $\bar{p} d \rightarrow p \pi^{-}$and $\bar{p} d \rightarrow \Sigma^{-} K^{+}\left(\right.$and/or $\left.\Sigma^{0} K^{0}, \Lambda K^{0}\right)$ are desirable. With this 
assumption, one can analyze strange particle production and compare it to the expected yield in the presence of $B=0$ annihilations only, as explained in Sec. III. In our previous works, we did such an analysis in some simple cases. The difficulty in this analysis is a correct estimate of the factors $D_{B}$ [Eq. (3.1)]. For the interesting case of $\bar{p}-\mathrm{Ta}$ at $4 \mathrm{GeV} / c{ }^{7}$ we postpone this analysis to a forthcoming paper, where we will use the full complexity of the intranuclear cascade model. We here concentrate our attention on the factors $P_{B}$, which can be estimated by a geometrical model. We showed in Sec. III that annihila- tion on two nucleons or more are expected to occur with a frequency of the order of $10 \%$. It is however unreasonable to expect the appearance of an annihilating system involving really many nucleons at the same time, as it was suggested in Ref. 38.

Let us finally emphasize that another check of the relevance of our approach is provided by the mesonless annihilations, and in particular by the $\bar{n}^{3} \mathrm{He} \rightarrow p p$ reaction. We predict a non-negligible yield, which could be measurable in the near future.
${ }^{1}$ B. M. Pontecorvo, Zh. Eksp. Teor. Fiz. 30, 947 (1956) [Sov. Phys. - JETP 3, 966 (1957)].

2J. Rafelski, in Physics at LEAR, edited by U. Gastaldi and R. Klapisch (Plenum, New York, 1984).

${ }^{3}$ S. Kahana, Proceedings of the Workshop Physics at LEAR, edited by U. Gastaldi and R. Klapisch (Plenum, New York, 1984), p. 495.

${ }^{4} J$. Cugnon and J. Vandermeulen, Phys. Lett. 146B, 16 (1984).

${ }^{5}$ J. P. Bocquet et al., Phys. Lett. B 182, 146 (1986).

${ }^{6} \mathrm{~J}$. P. Bocquet et al., Phys. Lett. B 192, 312 (1987).

${ }^{7}$ M. Miyano et al., Phys. Rev. Lett. 53, 1725 (1984).

${ }^{8}$ J. Cugnon and J. Vandermeulen, Phys. Rev. C 36, 2726 (1987).

${ }^{9}$ C. J. Hamer, Nuovo Cimento A 12, 162 (1972).

${ }^{10}$ S. J. Orfanidis and V. Rittenberg, Nucl. Phys. B59, 570 (1973).

${ }^{11} \mathrm{~J}$. Vandermeulen, Lett. Nuovo Cimento 11, 243 (1974).

${ }^{12}$ H. Möhring et al., Nucl. Phys. B85, 227 (1975).

${ }^{13}$ B. Margolis, W. J. Meggs, and N. Weiss, Phys. Rev. D 13, 2551 (1976).

${ }^{14} \mathrm{C}$. Dover, in Proceedings of the Third Conference on the Intersections Between Particle and Nuclear Particles, Rockport, 1988 (AIP, New York, to be published).

${ }^{15}$ R. Hagedorn and I. Montvay, Nucl. Phys. B59, 45 (1973).

${ }^{16}$ E. Fermi, Prog. Theor. Phys. 5, 570 (1950).

${ }^{17}$ R. Armenteros et al., CERN Report No. CERN/PSCC/80101, 1980).

${ }^{18}$ R. Hagedorn, I. Montvay, and J. Rafelski, in Hadronic Matter at Extreme Energy Density, edited by N. Cabibbo and L. Sertorio (Plenum, New York, 1980), p. 49.

${ }^{19}$ P. Gregory et al., Nucl. Phys. B102, 189 (1976).

${ }^{20} \mathrm{~J}$. Vandermeulen, Lett. Nuovo Cimento 28, 60 (1980).

${ }^{21}$ J. Vandermeulen, Lett. Nuovo Cimento 21, 33 (1978)

${ }^{22}$ H. Muirhead and P. Gregory, Antinucleon-Nucleon Interactions, edited by G. Ekspong and S. Nilsson (Pergamon, New
York, 1987), p. 331.

${ }^{23}$ F. Balestra et al., Nucl. Phys. A452, 573 (1986).

${ }^{24}$ A. Lejeune, P. Grangé, M. Martzolff, and J. Cugnon, Nucl. Phys. A453, 189 (1986).

${ }^{25}$ V. M. Chechetkin, M. Yu. Khlopov, M. G. Sapozhnikov, and Ya. B. Zeldovich, Phys. Lett. 118B, 329 (1982).

${ }^{26} \mathrm{H}$. Überall, Electron Scattering from Complex Nuclei, Part A (Academic, New York, 1971), Chap. 5.

${ }^{27}$ S. Ahmad et al., Physics with Antiprotons at LEAR in the Acol Era, edited by U. Gastaldi et al. (Editions Frontières, Gif sur Yvette, 1986), p. 353.

${ }^{28}$ G. A. Smith, in The Elementary Structure of Matter, edited by J. M. Richard et al. (Springer-Verlag, Berlin, 1988), p. 197.

${ }^{29}$ This corrects our estimate of Ref. 4, where an isospin factor had been neglected.

${ }^{30}$ J. Riedlberger et al. (ASTERIX Collaboration) Nucl. Phys. C (to be published).

${ }^{31}$ L. A. Kondratyuk and M. G. Sapozhnikov, Physics at LEAR with Low Energy Antiprotons, edited by C. Amsler et al. (Harwood-Academic, New York, 1988), p. 771.

${ }^{32}$ R. Bizzarri et al., Lett. Nuovo Cimento 2, 431 (1969).

${ }^{33}$ B. Y. Oh et al., Nucl. Phys. B51, 57 (1973).

${ }^{34}$ E. Oset, Physics at LEAR with Low Energy Antiprotons, edited by C. Amsler et al. (Harwood-Academic, New York, 1988), p. 753.

${ }^{35} \mathrm{~J}$. Cugnon, in The Elementary Structure of Matter, edited by J. M. Richard et al. (Springer-Verlag, Berlin, 1988), p. 211.

${ }^{36} \mathrm{~J}$. M. Richard, in Proceedings of the Third Conference on the Intersections Between Particle and Nuclear Particles, Rockport, 1988 (AIP, New York, to be published).

${ }^{37}$ Particle Data Group, Phys. Lett. 170B, 1 (1986).

38J. Rafelski, Phys. Lett. 207B, 371 (1988). 